\title{
An Enhanced Fast Handover with Low Latency for Mobile IPv6
}

\author{
Ruidong Li, Student Member, IEEE, Jie Li*, Senior Member, IEEE, Kui Wu, Member, IEEE, \\ Yang Xiao, Senior Member, IEEE, and Jiang Xie, Member, IEEE
}

\begin{abstract}
One of the most important challenges in Mobile IPv6 is to provide the service for a mobile node to maintain its connectivity to the Internet when it moves from one domain to another, which is referred to as handover. Here we deal with the fast handover problem, which is to provide rapid handover service for the delay-sensitive and real-time applications. In this paper, we propose an enhanced fast handover scheme for Mobile IPv6. In our scheme, each AR (Access Router) maintains a CoA (Care of Address) table and generates the new CoA for the MN that will move to its domain. At the same time, the binding updates to home agent and correspondent node are to be performed from the time point when the new $\mathrm{CoA}$ for MN is known by PAR (Previous AR). Also the localized authentication procedure cooperated with the proposed scheme is provided. For the comparison with the existing fast handover scheme, detailed performance evaluation is performed. From the evaluation results, we can see that the proposed enhanced fast handover scheme can achieve low handover latency and low packet delay.
\end{abstract}

Index Terms-Mobility, mobile IPv6, fast handover scheme, enhanced fast handover scheme, performance analysis.

\section{INTRODUCTION}

$\mathbf{T}$ O make a mobile node (MN) stay connected to the Internet regardless of its location, mobile IPv6 [11] is proposed as the next generation wireless Internet protocol. This is achieved primarily through using CoA (Care of Address) to indicate the location of the MN. Although the Mobile IPv6 protocol has many promising characteristics and presents an elegant mechanism to support mobility, it has an inherent drawback. That is, during a handover process, there is a short period that the mobile node is unable to send or receive packets because of link switching delay and IP protocol operations [12]. Handover is the process by which an MN keeps its connection active when it moves from one access medium to another [1]. The handover process happens when the $\mathrm{MN}$ moves from one access medium to another, and it should accomplish three operations: movement detection, new CoA configuration, and binding update (BU). During handover period, the $\mathrm{MN}$ is unable to send or receive packets as usual. The length of this period which is called handover latency is very critical for the delay-sensitive and real-time services.

Manuscript received August 14, 2006; revised October 11, 2006; accepted December 13, 2006. The associate editor coordinating the review of this paper and approving it for publication was Y.-B. Lin.

*Corresponding author is with the Graduate School of Systems and Information Engineering, University of Tsukuba, Tsukuba Science City, 3058573, Japan (e-mail: lijie@cs.tsukuba.ac.jp).

Digital Object Identifier 10.1109/TWC.2008.060582.
In the IPv6 specification [11], a common handover scheme has been proposed to support mobility. But the handover latency is so large that it is unbearable for many applications. To reduce the handover latency, two categories of protocols have been proposed. One focuses on the network architecture design [4], [7], [14], [16], [17], such as HMIP [17], IDMP [14]. The other focuses on the mechanism to reduce latency by $\mathrm{MN}$ and AR themselves, such as fast handover [2], [12], [13], [19]. In this paper, we focus on the design of fast handover scheme.

Fast handover scheme is the scheme to reduce the handover latency by anticipating handover and performing some operations prior to a break of the radio link. It includes two problems [2], [12], [13], [19]: how to allow an MN to send packets as soon as it detects a new subnet link, and how to deliver packets to a mobile node as soon as its attachment is detected by the new access medium. To solve these problems as much as possible, a fast handover scheme for Mobile IPv6 [12] has been proposed. In this scheme, the movement detection latency is reduced by providing the MN with the information about the new access point and the associated subnet prefix information when the $\mathrm{MN}$ is still connected to its current subnet. The new CoA (Care of Address) configuration latency is reduced by neglecting DAD (Duplicated Address Detection) [18] and by generating and configuring new CoA by $\mathrm{MN}$ itself. To reduce the binding update latency, a bidirectional tunnel between previous access router (PAR) and the new access router (NAR) is established.

In the fast handover scheme, it is noted that the DAD procedure may consume much time, especially if the DAD begins after the link is created to the new access router. If duplicate CoA occurs, the service quality for users will decrease greatly. At the same time, we note that the binding updates to the HA (Home Agent) and $\mathrm{CN}$ (Correspondent Node) are performed after the time point when the $\mathrm{MN}$ is IP-capable on the new subnet link [12]. Because of this, the MN communicates with the $\mathrm{CN}$ directly via the NAR without using tunnel in a very late time. Thus the packet delay for some packets sent during the handover will be enlarged.

Our work is motivated by the following questions: Can DAD procedure be performed beforehand? Does the binding update to $\mathrm{HA} / \mathrm{CN}$ need to be performed after the MN is IPcapable on the new subnet link? After investigation, we found that the nCoA (new CoA) generation and DAD procedure can be performed before handover starts. At the same time, we found that when $\mathrm{nCoA}$ is informed to PAR (Previous AR), 


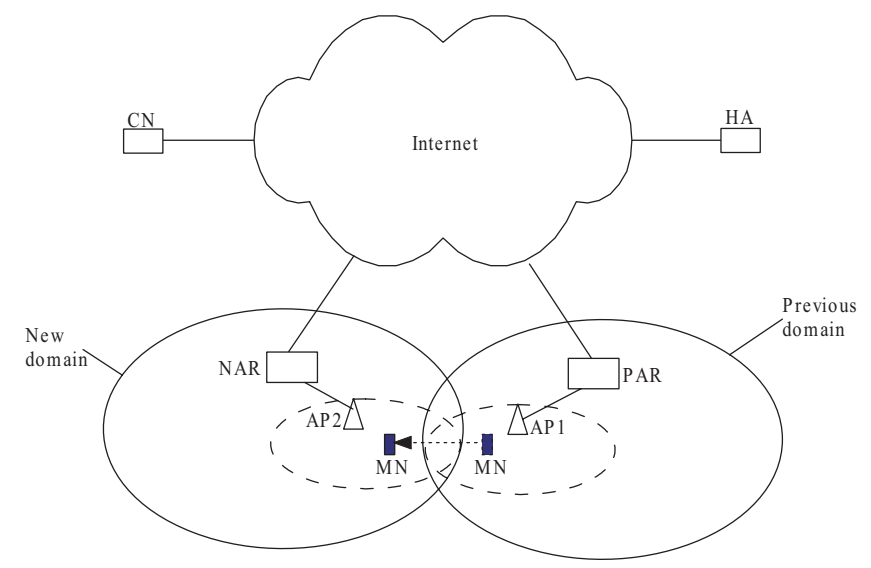

Fig. 1. System architecture for Mobile IPv6.

the handover to the new access point will definitely happen. Therefore, the binding update to the HA/CN can be performed at the time point when the new CoA is known by PAR.

In this paper, we propose a scheme for fast handover in Mobile IPv6. To reduce the latency in the DAD procedure, we let the new AR (Access Router) construct a new CoA, perform DAD for the MN and store this new CoA to the nCoA (new CoA) table when anticipating that a handover for an $\mathrm{MN}$ is about to happen. Then when the $\mathrm{MN}$ requests the nCoA through the previous AR, this new CoA will be distributed to the MN from the NAR via PAR. At the same time, to reduce the registration latency in the binding update, the binding update to the $\mathrm{HA} / \mathrm{CN}$ will be performed after the PAR knows the nCoA. Also the localized authentication procedure cooperated with the proposed scheme is provided. We perform detailed performance analysis on the proposed enhanced scheme. By the analysis, it is found that compared with the existing fast handover scheme, the handover latency and packet delay can be reduced by the proposed enhanced scheme.

The rest of the paper is organized as follows: In Section II, we will provide system descriptions. The fast handover for Mobile IPv6 is introduced in Section III. In Section IV, an enhanced fast handover scheme for Mobile IPv6 is proposed. We develop a comprehensive analytical model and provide careful performance evaluation in Section V. Finally, we conclude the paper in Section VI.

\section{SYSTEM DESCRIPTIONS}

Consider the network architecture depicted in Figure 1. There are three entities in the network architecture: HA (Home Agent), AR (Access Router), AP (Access Point). Besides these, there are two kinds of nodes, $\mathrm{CN}$ (Correspondent Node) and MN (Mobile Node). The MN connects to the Internet via $\mathrm{AP}$ and $\mathrm{AR}$.

To describe the handover scheme, we address the common situation where handover happens. There are two access points, AP1 and AP2, in the network, which connect to different access routers, PAR (Previous Access Router) and NAR (New Access Router), respectively. As the figure illustrates, when the MN moves from the radio region of the AP1 to that of the AP2, the handover happens.
When the handover induced by moving between different domains happens, the MN should perform layer 2 handover and Layer 3 Handover. Layer 2 handover is the process with which the mobile node changes from one access point to another, here from AP1 to AP2. Layer 3 handover is the process that the MN changes the attachment from one access router to another, here from PAR to NAR. The layer 3 handover includes three operations: movement detection, new CoA configuration, and binding update. Thus the handover latency includes three parts:

- L2 handover latency, which is defined as the time interval from the moment that layer 2 link down trigger from the PAR happens to the moment that the layer 2 link up trigger to the NAR happens. From the measurement performed in [13], the link layer handover when using IEEE $802.11 \mathrm{~b}$ cards will be more than 1 second and less than 2 seconds depending on the different makers.

- Rendezvous time delay [20], which includes two kinds of latency. One is movement detection latency, which is the time interval taken by the MN to detect the presence of a NAR at the new access network, and the other is the new CoA configuration latency, which is the time interval taken to configure a new CoA for the MN.

- Registration delay, which is the time that it takes to send $\mathrm{BU}$ to the $\mathrm{HA} / \mathrm{CN}$ and the subsequent resumption of communications indicated by a new data packet arriving at the MN from the NAR without passing through the tunnel between NAR and PAR.

In this paper, we will not consider details on the layer 2 handover, and treat it as a fixed time interval consumed.

Additionally, the link layer handover triggers [2], [13] are utilized in this paper. These triggers are signals related to the link layer handover process. The first trigger is an early notice of an upcoming change in the link layer point of attachment of the $\mathrm{MN}$, referred to as handover anticipation trigger. The second trigger, the layer 2 link up trigger occurs when the link between the $\mathrm{MN}$ and the new access medium is established. It is an indication that layer 3 can now send packets over the link. All layer 2 activities in configuring the link are expected to be complete at this point. The third trigger, the layer 2 link down trigger, indicates that the link between the $\mathrm{MN}$ and the old access medium is lost, which means that layer 3 cannot send any more packets over this link.

\section{FASt HANDOver Scheme For Mobile IPv6}

To reduce the handover latency occurred in Mobile IPv6, fast handover schemes for Mobile IPv6 are proposed in [12]. In [12], two schemes are proposed: one is the predictive fast handover scheme and the other is the reactive fast handover scheme. The main difference between these two schemes is on the time to establish the tunnel between the PAR and NAR. By the predictive handover, the tunnel is established before layer 2 handover, but by the reactive handover, the tunnel is established directly after layer 2 handover. Figure 2 shows the packet flows for the predictive fast handover scheme. Figure 3 shows the timing graph for the predictive fast handover scheme. We focus on the predictive fast handover scheme in this paper because it has shorter latency than the reactive one. 


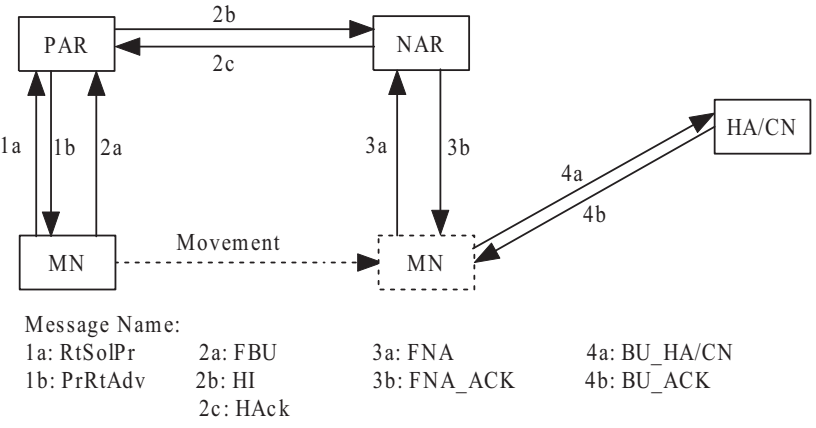

Fig. 2. Packet flows for the predictive fast handover scheme for Mobile IPv6.

We note that the DAD is neglected when the new CoA configuration is performed in the fast handover scheme. Although the probability that the duplicate address exists is small, this procedure cannot be neglected. If the DAD is not performed at the beginning and is performed after the node has set up a link to the new AP, much time will be wasted if duplicate address exists and such latency cannot be tolerated for latencysensitive applications.

On the other hand, we notice that the binding update and registration procedure cannot be started as soon as possible. By the fast handover scheme, the binding updates to HA/CN are performed after the link is created to the new access medium. After investigation, we find that when the handover starts and the $\mathrm{nCoA}$ is known by PAR, the handover procedure will definitely be performed even if there are duplicated addressed or some other situations. Therefore, using this discovery, we propose performing the $\mathrm{BU}$ to $\mathrm{HA} / \mathrm{CN}$ beforehand.

\section{Proposed Enhanced Fast Handover Scheme For MoBILE IPv6}

Based on the above considerations, we propose an enhanced fast handover scheme for Mobile IPv6, which includes two parts. One is the new CoA generation maintenance method. By this method, the new CoA is generated by NAR beforehand and NAR maintains a CoA table for communications. The other is the proposed enhanced fast handover scheme. In this scheme, the binding updates to $\mathrm{HA} / \mathrm{CN}$ are brought beforehand.

\section{A. Proposed New CoA Generation and Maintenance Method}

1) Method Overview: Here a new CoA generation and maintenance method is proposed to reduce the DAD (Duplicate Address Detection) latency in fast handover scheme, where the new CoA is generated by NAR instead of MN and the DAD is performed beforehand. It is also important to note that except for the reduction on latency, bringing the DAD procedure forward can make the issued nCoA unique. Thus, the binding update to $\mathrm{HA} / \mathrm{CN}$ can be brought forward because there is no problem on the $\mathrm{nCoA}$.

We note that there are two kinds of new CoA generation methods [18]. One is the stateless autoconfiguration method and the other is the stateful autoconfiguration method. In this paper, we only consider stateless autoconfiguration method

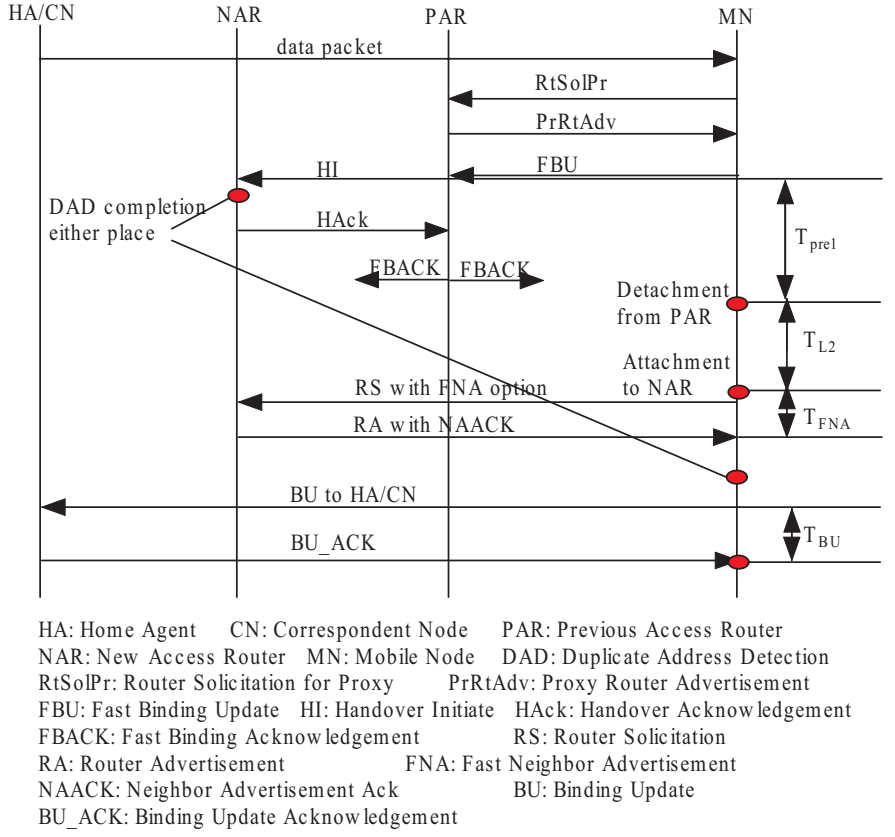

Fig. 3. Timing graph for the fast handover scheme.

since it is widely used in Mobile IPv6. Brief descriptions are provided as follows. Firstly the link-local address is generated and then the DAD is performed. If DAD fails, the manual configuration should be carried out. If there is no problem with the DAD process, the router prefix should be obtained and the new CoA is generated using the link-local address and the router prefix.

Though the probability that the duplicate link-local address occurs on the same link is low, it still cannot be ignored because communication quality will be greatly influenced if the duplicate address occurs. In the fast handover scheme [12], the DAD procedure is performed after the NAR receives the handover initiate packet or creating a link to the NAR is finished as in Figure 3. Both methods will bring unpredictable latency.

To reduce this latency, it is more reasonable if the new CoA is generated and the DAD is performed by NAR before requesting the nCoA by MN. At the same time, the information held by MN and needed for address autoconfiguration is the interface of MN. Thus if the NAR can know the interface and the AP-ID of the NAP, the NAR can autoconfigure the new CoA instead of the MN. Thus the new CoA generation operation can be moved from the MN to NAR. At the same time, the NAR can perform the DAD by searching the CoA list. Therfore, in the enhanced scheme, the unpredictable delay related to DAD can be avoided.

2) Method Operations: In the proposed enhanced scheme, we let each AR maintain a CoA table as Table 1. This table is used to store the currently used CoA and the new CoAs for the coming MNs. If the CoA is a currently used one, the "Active" label should be specified as T. Otherwise, it will be specified as $\mathrm{F}$, because this is an inactive $\mathrm{CoA}$ for a coming MN. Besides the "Active" label, there are some other options, such as "Start Time," "Valid Time."

When an MN anticipates that the handover to a new domain 
TABLE I

AN EXAMPLE OF COA TABLE

\begin{tabular}{|c|c|c|c|c|c|}
\hline MN-ID & PAR & nCoA & Active & Start Time & Valid Time \\
\hline 13 & PAR1 & $\ldots$ & F & $10: 20$ & 200 s \\
\hline 25 & PAR2 & $\ldots$ & T & $21: 02$ & $200 \mathrm{~s}$ \\
\hline
\end{tabular}

is about to happen, that is, the handover anticipation trigger occurs, the following steps should be performed.

Step 1: The PAR should send the related information of this MN $[P A R$, interface of $M N]$ to the NAR. Because the MN is currently in the domain of PAR, the PAR can have this information of the MN.

Step 2: After the NAR receives this message from the PAR, it will generate a new $\mathrm{CoA}$ for this MN using the received information about the MN. Then it will check whether this new CoA has been distributed to other MN through the DAD process. If this DAD process fails, a new CoA will be generated directly by the AR without using the information of the MN. If this DAD process succeeds, the generated new CoA is a valid one. Whatever which process has been performed, a valid new CoA has been obtained by the NAR.

Step 3: This new CoA will be inserted into the CoA table of NAR. In the mean time, the Active label should be set as F. Once this new CoA is activated by the link up trigger of this MN, this Active label will be set as $\mathrm{T}$.

\section{B. The Proposed Enhanced Fast Handover Scheme}

1) Scheme Overview: To reduce the handover latency, we merge the movement detection procedure and tunnel establishment procedure, and let PAR send $B U_{-} H A / C N$ (Binding Update to $\mathrm{HA}$ and $\mathrm{CN}$ ) directly after PAR knows the new CoA. Also the DAD will not be performed during the period when the tunnel is established as in the predictive fast handover scheme, because DAD has already been performed beforehand. Thus the latency for performing DAD can be reduced.

In the existing fast handover scheme, the binding update to the HA/CN is performed after attaching to the NAR. However, in the proposed enhanced scheme, the binding update to the $\mathrm{HA} / \mathrm{CN}$ is brought forward to the time before the layer 2 handover. Thus in our scheme, the completion time of BU to HA/CN is advanced and the tunnel between PAR and NAR will exist shorter than in the fast handover scheme. Therefore, many packets passing to the MN through the tunnel can be sent to the MN directly without using the tunnel. That means the latency for these packets can be reduced.

Note that in our scheme, binding update is performed by PAR not NAR, because it should be guaranteed that the nCoA has been known by PAR. That is, if PAR has not received any information on $\mathrm{nCoA}$ but binding update has been performed, it will lead to topology error because the moving $\mathrm{MN}$ has two CoAs at the same time. On the other hand, after PAR knows the nCoA, it will know that the MN has moved to the domain of NAR and make the current CoA unavailable
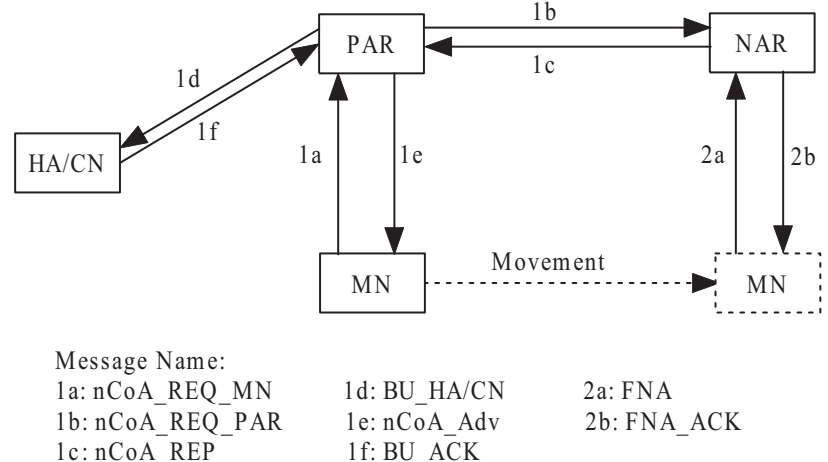

Fig. 4. Packet flows for the enhanced fast handover scheme.

to communicate with the MN directly. At the same time, the $\mathrm{nCoA}$ is available to the corresponding nodes and HA by binding update. Therefore, there will be no problem on routability test after the $\mathrm{HA} / \mathrm{CN}$ receives $B U_{-} H A / C N$ for the reason that there is only one $\mathrm{CoA}$ is in use for the $\mathrm{MN}$ at one time.

Also we notice that even DAD procedure has not been brought forward as proposed, the binding update procedure can still be performed by PAR at the time point when the nCoA is known by PAR. What we need to do is to label this nCoA as a new one for an MN that will move from PAR to NAR. Thus even if the DAD fails at the beginning, the NAR still can identify the packets which are directed to the specified MN because the NAR can differentiate the packet destination by the information of $\mathrm{nCoA}$, PAR and MN.

2) Scheme Operations: The packet flow for the enhanced fast handover scheme is depicted in Figure 4.

In the following, the operations for the proposed enhanced fast handover scheme will be given.

Step 1: Firstly the MN will send the "new CoA Request from MN ( $\left.n C o A_{-} R E Q_{-} M N\right)$ " to PAR. After PAR receiving this message, it sends out the "New CoA Request from PAR ( $\left.n C o A_{-} R E Q_{-} P A R\right)$ " including the information of PAR and MN to NAR. Then NAR will find the right CoA for this MN from the CoA table and send back a "new CoA Reply ( $n C o A_{-} R E P$ )" including the new CoA to the PAR. Once receiving $n C o A_{-} R E P$ from NAR, the PAR will advertise this nCoA to the MN by " $n C o A_{-} A d v$ " message. At the same time, the "Binding Update to HA/CN ( $\left.B U_{-} H A / C N\right)$ " messages are sent to HA and $\mathrm{CN}$ separately by PAR. Also during binding update procedure, the "Binding Update Acknowledgement ( $\left.B U_{-} A C K\right)$ " should be replied by $\mathrm{HA} / \mathrm{CN}$.

Step 2: After the MN moves to the new domain of NAR, it will send a router solicitation with the FNA option. Then NAR will reply with a router advertisement with the $F N A_{-} A C K$ option.

To describe the enhanced scheme clearly, the detailed timing graph for the enhanced scheme is provided in Figure 5. 


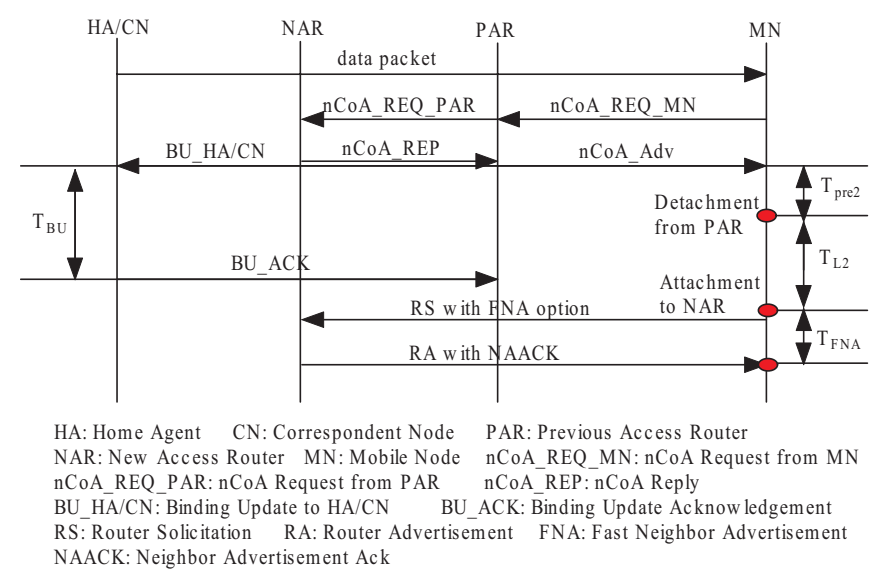

Fig. 5. Timing graph for the proposed enhanced fast handover scheme.

\section{Security Discussion}

Security is not the focus of this paper. But we would like to provide some methods to cooperate our enhanced scheme. When one user moves from one domain to another, the authentication to the MN from HA should be performed [3], [6], [8], [9], [10], [15]. Currently, challenge/response [15] mechanism attracts much more attention as the method for authenticating the $\mathrm{MN}$ in the foreign domain. When MN moves out of home domain and arrives at the first foreign domain, MN will perform challenge/response mechanism to make its identity verified by HA. Thus, we can assume that the PAR has established the trust relations including mutual security association with the MN. That is, the PAR knows the security association related to the MN. After entering the first foreign domain, the authentication procedure for entering other foreign domains can be performed as follows. Before PAR sends $n C o A_{-} R E Q_{-} P A R$ to NAR, it generates a random number and appends this number and the information of the security association related to this $\mathrm{MN}$ to $n C o A_{-} R E Q_{-} P A R$ message. Then it sends this message to NAR. At the same time, PAR appends the generated random number to $n C o A_{-} A d v$ and send it to the $\mathrm{MN}$. We assume that the general security is assured. That is, all the messages sent are encrypted by symmetric cryptography. Then when the MN moves to the NAR, it will only send this random number to the NAR to verify its identity. In the mean time, they can use the security association known by NAR to communicate with each other. Here, the authentication delay can be reduced greatly, because in challenge/response mechanism, the MN should be authenticated by HA every time when the MN moves to another domain. However, by our method, security association establishment can be carried out beforehand and the authentication procedure can be performed locally.

\section{Performance Evaluation}

Here we conduct performance evaluation to compare the enhanced fast handover scheme with the existing scheme [12]. It is obvious that reducing the handover latency is the goal of fast handover. On the other hand, packet delay can be used to reflect the service quality for users. Thus we consider the following two metrics:
- Latency of handover, denoted by LT and defined as the time interval from the moment that the packets cannot be sent or received to the moment that the mobile node can communicate with the $\mathrm{CN}$ directly via the NAR without using tunnel.

- Packet delay, denoted by PD and defined as the time interval from the moment that the packet is sent by the $\mathrm{CN}$ to the moment that the packet is received by $\mathrm{MN}$ during the handover.

In this paper, we design an analytical model to compare the above two metrics. We only focus on the predictive fast handover because it needs less time to perform handover than the reactive one since the tunnel between PAR and NAR by the predictive scheme is established before layer 2 handover [12]. The network architecture used for analysis is presented in Figure 1.

For convenience, the notations and basic assumptions are given as follows:

- Time 0: The time point that detachment from PAR occurs is 0 .

- $T_{L 2}$ : The layer 2 handover latency which is the time required to process $\mathrm{L} 2$ handover. It is defined as the time interval from the moment that the $\mathrm{MN}$ detaches from PAR to the moment that the MN attaches to the NAR.

- $T_{B U}$ : The binding update latency which is the time required to perform binding update to $\mathrm{HA} / \mathrm{CN}$. It is defined as the time interval from the moment that the binding updates to $\mathrm{HA} / \mathrm{CN}$ are sent to $\mathrm{HA} / \mathrm{CN}$ to the moment that the binding acknowledgements are received by MN.

- $T_{F N A}$ : The time to perform fast neighbour advertisement.

- $T_{D A D}$ : The time required for completing DAD process.

- $T_{\text {arrival }}$ : The time that the packet arrives at the PAR based on Time 0.

- $T(A, B)$ : The time required for a packet to pass from $A$ to $B$. For example, $T(M N, P A R)$ denotes the time required for a packet to pass from the MN to PAR. It is assumed that $T(A, B)=T(B, A)$.

- $T(A)$ : The time required for a packet to be processed at $A$. For example, $T(N A R)$ represents the time required for a packet to be processed (de-capsulated) at NAR.

The performance analysis on the above two metrics is given as follows.

\section{A. Latency of Handover}

Firstly, we investigate the time point when the packets cannot be sent or received. For the predictive fast handover scheme, the layer 2 handover can start at any time after fast binding update packet is received by PAR. Thus the time point that the packets cannot be sent and received in fast handover will be the time when the fast binding update packet is received by PAR. For our enhanced scheme, the packet forwarding will be stopped at the time point that $n C o A_{-} R E P$ is received by PAR.

Then, we investigate when the mobile node can communicate with the $\mathrm{CN}$ directly via the NAR without using tunnel. The completion time point is when both the binding update to $\mathrm{HA} / \mathrm{CN}$ and handover process have been finished. 
Figure 3 illustrates the latency for the fast handover in Mobile IPv6. It is assumed that the DAD process can be finished by NAR after receiving handover initiate packet before sending handover acknowledge packet because it is the best situation for the fast handover scheme. From Figure 3, we obtain that the handover latency includes four parts: $T_{p r e 1}$, $T_{L 2}, T_{F N A}, T_{B U}$, where $T_{p r e 1}$ is the time interval from the time point that packets cannot be sent and received to the time point that the MN detaches from the PAR, and $T_{L 2}, T_{F N A}$ and $T_{B U}$ are the time consumed to perform layer 2 handover, fast neighbor advertisement and binding update to HA/CN, respectively. Therefore we can obtain the handover latency for fast handover as follows.

$$
L T_{F H}=T_{p r e 1}+T_{L 2}+T_{F N A}+T_{B U} .
$$

From Figure 3, we can see that $T_{p r e 1}$ includes the time consumed for packet transmission from PAR to NAR, NAR to PAR, PAR to MN and the time for DAD process. Thus, we can obtain:

$$
\begin{aligned}
T_{\text {pre } 1} & =T(P A R, N A R)+T_{D A D}+T(N A R, P A R) \\
& +T(P A R, M N) \\
& =T(M N, P A R)+2 T(P A R, N A R)+T_{D A D} .
\end{aligned}
$$

Also obviously, $T_{F N A}$ includes the time consumed for packet transmission from MN to NAR and NAR to MN.

$$
\begin{aligned}
T_{F N A} & =T(M N, N A R)+T(N A R, M N) \\
& =2 T(M N, N A R) .
\end{aligned}
$$

Figure 5 illustrates the latency for the proposed enhanced fast handover scheme for Mobile IPv6. From Figure 5, we can see that the handover latency will be the maximum of $T_{\text {pre } 2}+T_{L 2}+T_{F N A}$ and $T_{B U}$. Therefore, we can obtain the handover latency for the proposed scheme as below:

$$
L T_{\text {Proposed }}=\max \left\{T_{\text {pre } 2}+T_{L 2}+T_{F N A}, T_{B U}\right\} .
$$

Similarly as (2) and (3), we can obtain:

$$
\begin{aligned}
T_{\text {pre } 2} & =T(P A R, M N) \\
T_{F N A} & =2 T(M N, N A R) .
\end{aligned}
$$

From the above discussions, we can see that $T_{\text {pre } 2}$ is not larger than $T_{\text {pre } 1}$. To perform comparison, there are two cases we should consider.

- Case 1: $T_{B U}<=T_{p r e 2}+T_{L 2}+T_{F N A}$

In this case, from (1) and (4), we can get:

$$
\begin{aligned}
L T_{F H} & =T_{\text {pre } 1}+T_{L 2}+T_{F N A}+T_{B U} \\
L T_{\text {Proposed }} & =T_{\text {pre } 2}+T_{L 2}+T_{F N A} .
\end{aligned}
$$

Time 0 is assumed to be the detachment from PAR. Thus we can obtain the latency comparison as Figure 6. From Figure 6, we can see that the proposed enhanced fast handover scheme can reduce the handover latency compared with the existing fast handover scheme in case 1.

- Case 2: $T_{B U}>T_{p r e 2}+T_{L 2}+T_{F N A}$ In this case, similarly we can obtain:

$$
\begin{aligned}
L T_{F H} & =T_{\text {pre } 1}+T_{L 2}+T_{F N A}+T_{B U} \\
L T_{\text {Proposed }} & =T_{B U} .
\end{aligned}
$$

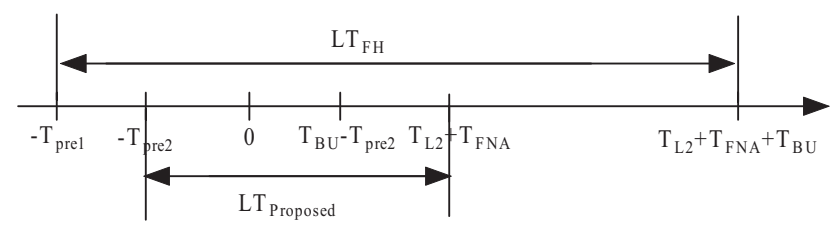

Fig. 6. Handover latency comparison in Case 1.

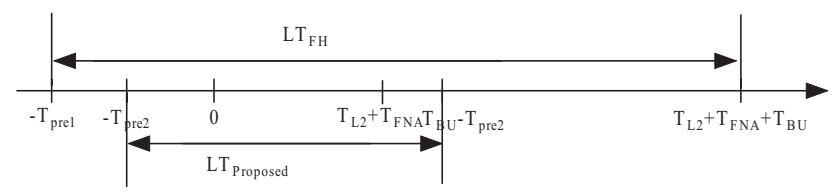

Fig. 7. Handover latency comparison in Case 2.

We can get the latency comparison as Figure 7. From Figure 7, we can see that the latency for handover can be improved by the proposed enhanced fast handover scheme in case 2.

In conclusion, we obtain

$$
L T_{F H}>L T_{\text {Proposed }} .
$$

It means that the time required to complete the whole handover procedure will be reduced. The reason is that the DAD procedure has been performed previously and the binding update procedure has been brought forward. It will benefit much more for the handover latency when the security issues [3], [6], [8] for Mobile IPv6 are considered. In the enhanced fast handover scheme, it can register the new CoA to HA in advance. At the same time, the MN can be authenticated locally by NAR instead of far HA. Thus the handover latency can be reduced further because the authentication procedure is moved ahead.

\section{B. Packet Delay}

To investigate packet delay, we firstly consider the tunnel existing time. Tunnel existing time is defined as the time interval from the moment that the tunnel is established to the moment that the tunnel is destructed. According to [12], the tunnel remains active until the MN completes the binding update with its correspondents in the fast handover scheme. Thus the tunnel existing time equals to the handover latency described as above.

There are two types of packet flows in the network as in Figure 8, depending on whether the tunnel between PAR and NAR exists. In type (1), the packets flow from $\mathrm{CN}$, via PAR, NAR, and AP2, then reach MN. In type (2), the packets flow from CN, via NAR and AP2, then arrive at MN. If the tunnel exists, that is, before the binding updates have been finished, the packets are forwarded to the $\mathrm{MN}$ through the tunnel between the PAR and NAR as the packet flow in type (1) in Figure 8. Otherwise, the packets are forwarded to the MN via the NAR without passing through the tunnel as the packet flow in type (2) in Figure 8. Without loss of generality, we assume that the PAR and NAR have no difference. That is, the time required for a packet travelling from $\mathrm{CN}$ to PAR equals the time required for a packet travelling from $\mathrm{CN}$ to 


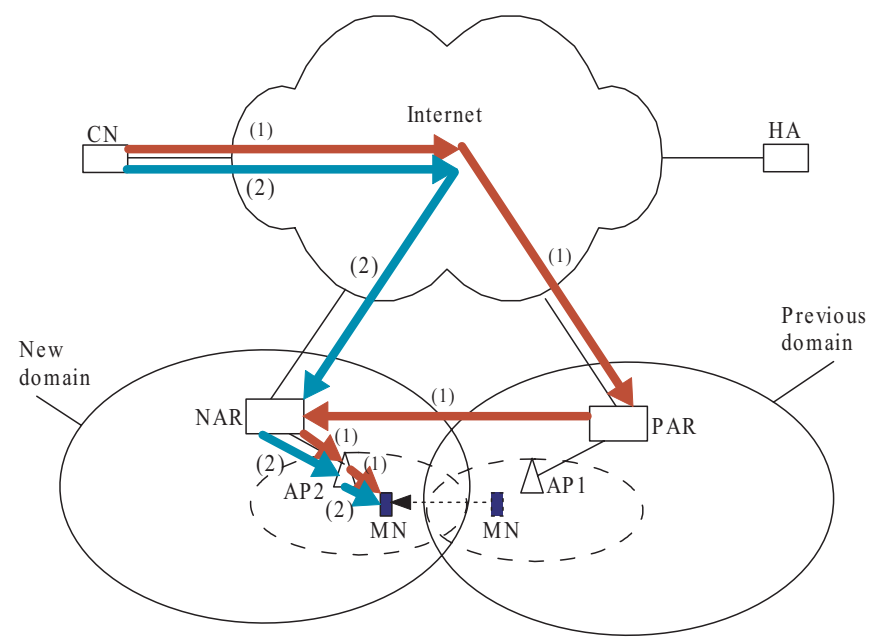

Fig. 8. Packet flows based on the time point when binding updates complete, the chocolate one is the type (1) of packet flow and the blue one is the type (2) of packet flow.

NAR. Thus, we can see that if the packets should be forwarded through a tunnel between PAR and NAR, the packet delay will be larger because of the encapsulation and decapsulation operations and the time required to forward packets from PAR to NAR.

For flow in type (1), the packet delay from $\mathrm{CN}$ to $\mathrm{MN}$ includes the time consumed for packet transmission from $\mathrm{CN}$ to PAR, PAR to NAR, NAR to MN, and the time consumed for processing at PAR and NAR. Thus we can obtain the packet delay for type (1) as follows:

$$
\begin{aligned}
P D 1 & =T(C N, P A R)+T(P A R)+T(P A R, N A R) \\
& +T(N A R)+T(N A R, M N) .
\end{aligned}
$$

For flow in type (2), similarly as (8), the packet delay for transmission from $\mathrm{CN}$ to $\mathrm{MN}$ will be

$$
P D 2=T(C N, N A R)+T(N A R, M N) .
$$

Without loss of generality, we assume that there is no difference between NAR and PAR. Thus we can get that $T(C N, N A R)=T(C N, P A R)$. Thus, we can obtain:

$$
P D 1-P D 2=T(P A R)+T(P A R, N A R)+T(N A R) .
$$

From here we analyze the packet delay. The packet delay can be differentiated by the time point that the fast neighbor advertisement has been completed. We compare the two schemes in two cases.

- Case 1: $T_{B U}<=T_{p r e 2}+T_{L 2}+T_{F N A}$.

For the fast handover scheme, during the time interval $\left[-T_{\text {pre } 1}, T_{L 2}+T_{F N A}\right]$, the packets sent to MN will be cached at NAR. At the time point that $\mathrm{MN}$ attaches to NAR, which is $T_{L 2}+T_{F N A}$, these packets will be sent to MN. Therefore, including the transmission time from $\mathrm{CN}$ to PAR and NAR to MN, the packet delay will be

$$
\begin{aligned}
P D_{F H 11} & =T(C N, P A R)+T_{L 2}+T_{F N A} \\
& +T(N A R, M N)-T_{\text {arrival }} .
\end{aligned}
$$

During the time interval $\left[T_{L 2}+T_{F N A}, T_{L 2}+T_{F N A}+\right.$ $\left.T_{B U}\right]$, the packets will be sent as flow in type (1). Thus

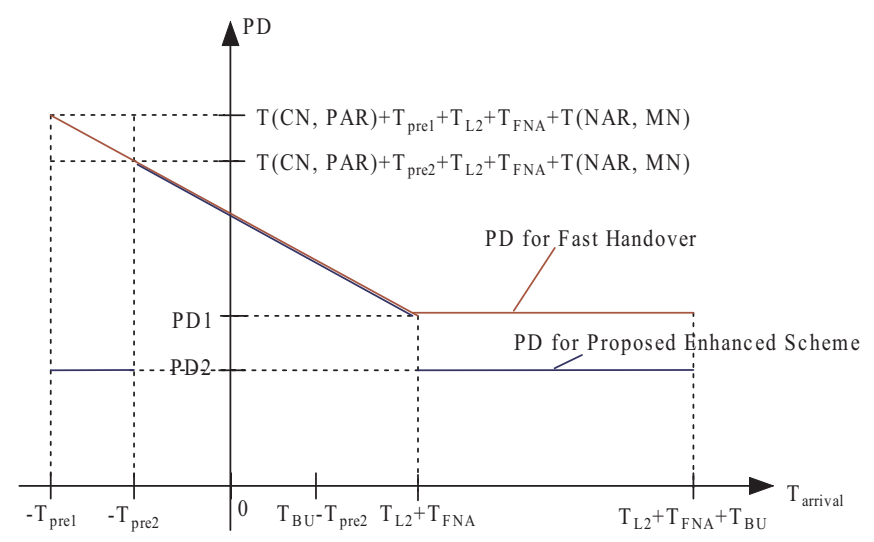

Fig. 9. PD comparison between the fast handover scheme and the proposed enhanced fast handover scheme in case 1 .

we can obtain the packet delay as follows.

$$
P D_{F H 12}=P D 1 .
$$

For the enhanced scheme, similarly as (11), during the time interval $\left[-T_{p r e 2}, T_{L 2}+T_{F N A}\right]$, the packet delay will be

$$
\begin{aligned}
P D_{\text {Proposed } 11} & =T(C N, P A R)+T_{L 2}+T_{F N A} \\
& +T(N A R, M N)-T_{\text {arrival }} .
\end{aligned}
$$

During the time interval $\left[T_{L 2}+T_{F N A}, T_{L 2}+T_{F N A}+\right.$ $\left.T_{B U}\right]$, the packet will be sent as flow in type (2). Thus, the packet delay will be

$$
P D_{\text {Proposed } 12}=P D 2 .
$$

The comparison for two schemes in Case 1 is provided in Figure 9. From the figure, we can see that the packet delay for the packets arriving at PAR at the time interval $\left[T_{L 2}+T_{F N A}, T_{L 2}+T_{F N A}+T_{B U}\right]$ can be improved by $P D 1-P D 2$ by the enhanced fast handover scheme.

- Case 2: $T_{B U}>T_{p r e 2}+T_{L 2}+T_{F N A}$.

We can obtain the packet delay in this case similarly as those in Case 1.

For the fast handover scheme, during the time interval $\left[-T_{\text {pre } 1}, T_{L 2}+T_{F N A}\right]$, the packet delay will be

$$
\begin{aligned}
P D_{F H 21} & =T(C N, P A R)+T_{L 2}+T_{F N A} \\
& +T(N A R, M N)-T_{\text {arrival }} .
\end{aligned}
$$

During the time interval $\left[T_{L 2}+T_{F N A}, T_{L 2}+T_{F N A}+\right.$ $\left.T_{B U}\right]$, the packet delay will be

$$
P D_{F H 22}=P D 1 \text {. }
$$

For the enhanced scheme, during the time interval $\left[-T_{p r e 2}, T_{L 2}+T_{F N A}\right]$, the packet delay will be

$$
\begin{aligned}
P D_{\text {Proposed } 21} & =T(C N, P A R)+T_{L 2}+T_{F N A} \\
& +T(N A R, M N)-T_{\text {arrival }} .
\end{aligned}
$$

During the time interval $\left[T_{L 2}+T_{F N A}, T_{B U}-T_{p r e 2}\right]$, the packet delay will be

$$
P D_{\text {Proposed } 22}=P D 1 .
$$




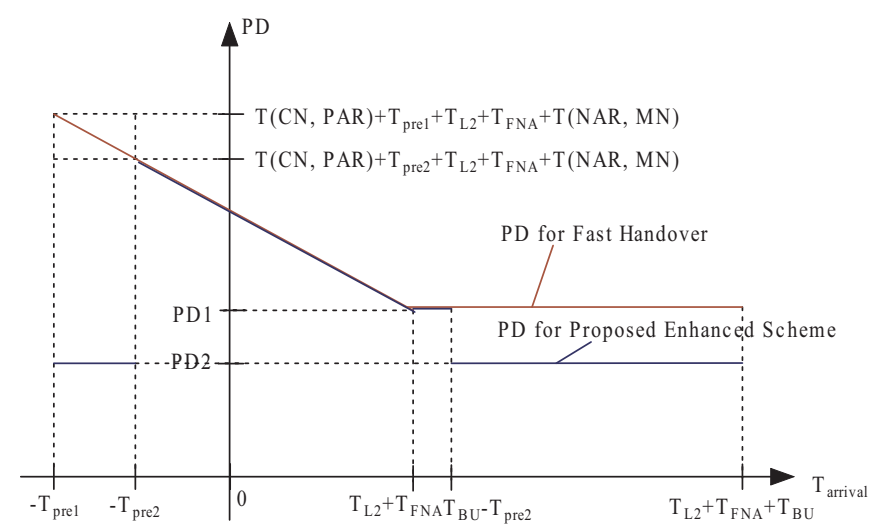

Fig. 10. PD comparison between the fast handover scheme and the proposed enhanced fast handover scheme in case 2 .

During the time interval $\left[T_{B U}-T_{p r e 2}, T_{L 2}+T_{F N A}+\right.$ $\left.T_{B U}\right]$, the packet delay will be

$$
P D_{\text {Proposed } 23}=P D 2 .
$$

The comparison for two schemes in case 2 is provided in Figure 10. From the figure, we can see that the packet delay for the packets arriving at PAR at the time interval $\left[T_{B U}-T_{p r e 2}, T_{L 2}+T_{F N A}+T_{B U}\right]$ can be improved by $P D 1-P D 2$ by the enhanced fast handover scheme.

Therefore, we can see that the packet delay for some packets can be reduced by $P D 1-P D 2$, which equals $T(P A R)+T(P A R, N A R)+T(N A R)$, since the binding updates $\mathrm{HA} / \mathrm{CN}$ can be performed in a faster fashion.

\section{Case Study}

Herein we study the percentage of packet delay which can be reduced by the enhanced scheme.

Because the reduced packet delay $P D 1-P D 2$ is larger than $T(P A R, N A R)$, we investigate the metric as minimum reduced packet delay, which is defined to be $T(P A R, N A R)$. In IPv6, it requires that every link in the Internet have an maximum transmission unit (MTU) of 1280 bytes or greater [5]. It is assumed that the link capacity is $2 \mathrm{Mbps}$ and the MTU for the IPv6 packets varies from 1028 bytes to 16384 bytes. Thus we can obtain the results as in Figure 11. From Figure 11, we can see that if the MTU is 1280 bytes, the minimum reduced packet delay is about 5 milliseconds; if the MTU is 16384 bytes, the minimum reduced packet delay reaches around 65 milliseconds. Additionally, if considering the encapsulation and decapsulation time and propagation delay on the router, we can see that $P D 1-P D 2$ is larger than the minimum reduced packet delay showed in Figure 11.

In addition, we investigate how the improved ratio changes with the number of hops between $\mathrm{CN}$ and PAR. The improved ratio is defined as $\frac{P D 1-P D 2}{P D 1}$, which is used to show the extent that our proposed enhanced handover scheme can reduce the packet delay compared with the fast handover scheme. It is assumed there are $\mathrm{N}$ hops between $\mathrm{CN}$ and PAR and the packet transmission delay on one hop is t. For example, $T(P A R, N A R)=t$. Because there is an AP between AR

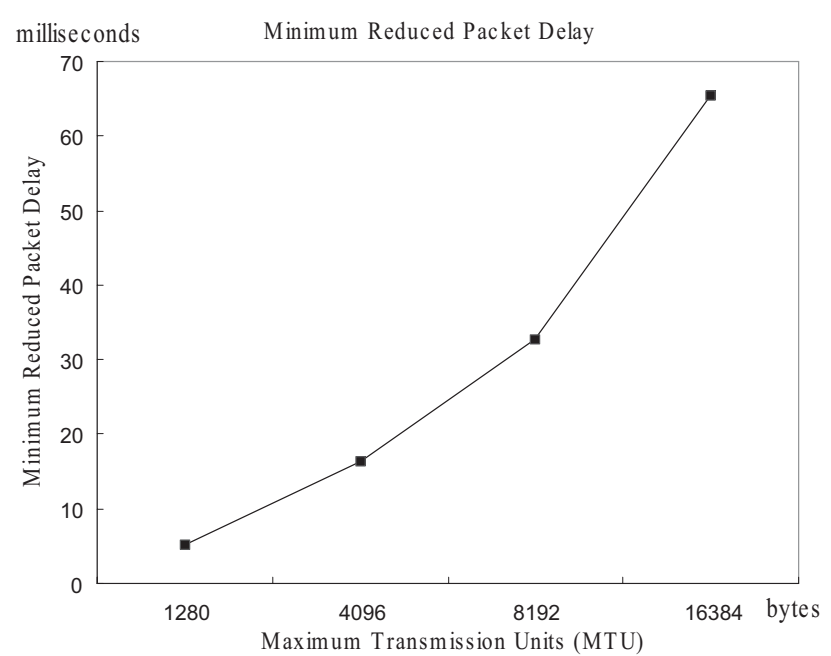

Fig. 11. The minimum reduced packet delay.

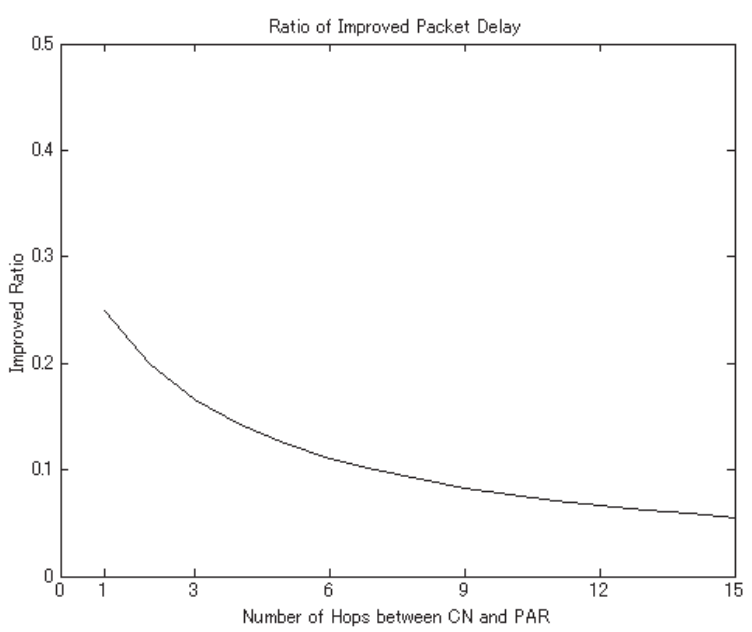

Fig. 12. The ratio of improved packet delay for the proposed enhanced fast handover scheme.

and $\mathrm{MN}$, there are two hops between NAR and MN. Thus, the enhanced scheme can improve packet delay by:

$$
\begin{array}{r}
\frac{P D 1-P D 2}{P D 1}=\frac{T(P A R)+T(P A R, N A R)+T(N A R)}{P D 1} \\
>\frac{T(P A R, N A R)}{T(C N, P A R)+T(P A R, N A R)+T(N A R, M N)} \\
=\frac{t}{(N+3) * t}=\frac{1}{N+3} .
\end{array}
$$

Figure 12 provides the ratio of reduced packet delay for the enhanced scheme. From this figure, we can see that if the number of hops between HA and PAR is 1, the improved ratio for packet delay can be larger than $25 \%$, and even the number of hops reaches 15 , the improved ratio still can be larger than $5.6 \%$.

\section{CONCLUSIONS}

In this paper, we propose an enhanced fast handover scheme for Mobile IPv6 and the collaborated security mechanism. In our scheme, each AR maintains a CoA table, and generates a 
new CoA for the MN that is anticipated to move to its domain. At the same time, we propose that binding updates to $\mathrm{HA} / \mathrm{CN}$ are performed by PAR from the time point when the $\mathrm{nCoA}$ is known by PAR. Also, in the proposed scheme, PAR provides security association related to $\mathrm{MN}$ and authentication random number to NAR. Thus the authentication procedure is advanced and localized. Finally, we analyze the handover delay and the packet delay for the proposed enhanced fast handover scheme. The analysis shows that with the proposed enhanced scheme, the handover latency can be reduced compared to the existing fast handover scheme. In the mean time, the packet delay for some packets sent in specified time intervals can be reduced by our scheme.

\section{REFERENCES}

[1] I. F. Akyildiz, J. Xie, and S. Mohanty, "A survey of mobility management in next-generation all-IP-based wireless systems," IEEE Wireless Commun., Aug. 2004.

[2] C. Blondia, O. Casals, L. Cerda, N. Wijngaert, and G. Willems, "Performance evaluation of Layer 3 low latency handoff mechanisms," Mobile Networks and Applications, vol. 9, no. 6, pp. 633-645, Dec. 2004.

[3] P. Calhoun, J. Loughney, E. Guttman, G. Zorn, and J. Arkko, "Diameter base protocol," draft-ietf-aaa-diameter-17.txt, Dec. 2002.

[4] A. T. Campbell, J. Gomez, S. Kim, A. G. Valko, C-Y. Wan, and Z. R. Turanyi, "Design, implementation, and evaluation of cellular IP," IEEE Personal Commun., pp. 42-49, Aug. 2000.

[5] S. Deering and R. Hinden, "Internet Protocol, version 6 (IPv6) specification," RFC 2460, Dec. 1998

[6] S. Glass, T. Hiller, S. Jacobs, and C. E. Perkins, "Mobile IP authentication, authorization, and accounting requirements," RFC 2977, Oct. 2000.

[7] E. Gustafsson, A. Jonsson, and C. E. Perkins, "Mobile IPv4 regional registration," Internet draft, IETF, draft-ietf-mobileip-reg-tunnel-09.txt, June 2004, work in progress.

[8] D. Harkins and D. Carrel, "The Internet key exchange (IKE)," RFC 2409, Nov. 1998.

[9] R. Housley, W. Ford, T. Polk, and D. Solo, "Internet X.509 public key infrastructure certificate and CRL profile," RFC 2459, Jan. 1999.

[10] S. Jacobs and S. Belgard, "Mobile IP public key based authentication," draft-jacobs-mobileip-pki-auth-02.txt, Mar. 1999.

[11] D. Johnson, E. C. Perkins, and J. Arkko, "Mobility support in IPv6," RFC 3775, June 2004.

[12] R. Koodli, "Fast handovers for mobile IPv6," RFC 4068, July 2005.

[13] K. Malki, P. Calhoun, T. Hiller, J. Kempf, P. McCann, A. Singh, H. Soliman, and S. Thalanany, "Internet draft: low latency handoffs in mobile IPv4," IETF, June 2002, work in progress.

[14] A. Misra, S. Das, A. Dutta, A. McAuley, and S. K. Das, "IDMPbased fast handoffs and paging in IP-based 4G mobile networks," IEEE Commun., vol. 40, no. 3, pp. 138-145, Mar. 2002.

[15] C. E. Perkins and P. Calhoun, "Mobile IPv4 challenge/response extensions," RFC 3012, Nov. 1999.

[16] R. Ramjee, K. Varadhan, L. Salgarelli, S. Thuel, S. Y. Wang, and T. La Porta, "HAWAII: a domain-based approach for supporting mobility in wide-area wireless networks," IEEE/ACM Trans. Networking, vol. 10, no. 3, pp. 396-410, June 2002.

[17] H. Soliman, C. Castelluccia, K. El Malki, and L. Bellier, "Hierarchical mobile IPv6 mobility management (HMIPv6)," RFC 4140, Aug. 2005.

[18] S. Thomson and T. Narten, "IPv6 stateless address autoconfiguration," RFC 2462, Dec. 1998.

[19] H. Velayos and G. Karlsson, "Techniques to reduce the IEEE 802.11b handoff time," in Proc. 2004 IEEE International Conference on Communications, vol. 7, June 2004.

[20] I. Vivaldi, B. Ali, H. Habaebi, V. Prakash, and A. Sali, "Routing scheme for macro mobility handover in hierarchical mobile IPv6 network," in Proc. 4th National Conference on Telecommunication Technology, 2003, pp. 88-92.

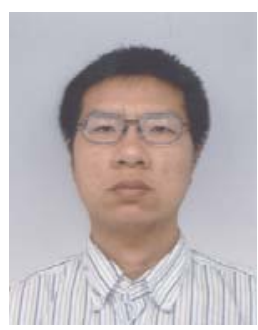

Ruidong $\mathbf{L i}$ received the B. S. degree from the Department of Information Science \& Electronic Engineering, Zhejiang University, Zhejiang, China, in 2001. Then, he went to University of Tsukuba, Tsukuba, Japan, and received M. S. degree there in 2005. He is currently a Ph.D. candidate at University of Tsukuba, Tsukuba, Japan. His research interests include ad hoc network, network security, seamless mobility, and modeling and performance evaluation.

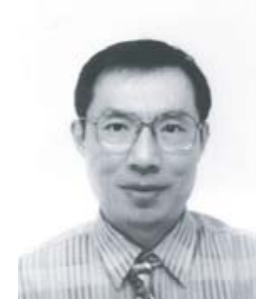

Jie Li received the B.E. degree in computer science from Zhejiang University, Hangzhou, China, in 1982, the M.S. degree in electronic engineering and communication systems from China Academy of Posts and Telecommunications, Beijing, China, in 1985. He received the Dr. Eng. degree from the University of Electro-Communications, Tokyo, Japan, in 1993. From 1985 to 1989, he was a research engineer in China Academy of Posts and Telecommunications, Beijing. From April 1993, he has been with the Department of Computer Science, Graduate School of Systems and Information Engineering, University of Tsukuba, Japan, where he is a Professor. His research interests are in mobile distributed multimedia computing and networking, OS, network security, modeling and performance evaluation of information systems. He received the best paper award from IEEE NAECON'97. He is a senior member of IEEE, and a member of ACM. He has served as a secretary for Study Group on System Evaluation of the Information Processing Society of Japan (IPSJ) and on the editorial boards for IPSJ (Information Processing Society of Japan) Journal, Information, and International Journal of High Performance Computing and Networking. He is also serving on Steering Committees of the SIG of System EVAluation (EVA) of IPSJ, the SIG of DataBase System (DBS) of IPSJ, and the SIG of MoBiLe computing and ubiquitous communications of IPSJ. He has also served on the program committees for several international conferences such as IEEE INFOCOM, IEEE GLOBECOM, and IEEE MASS.

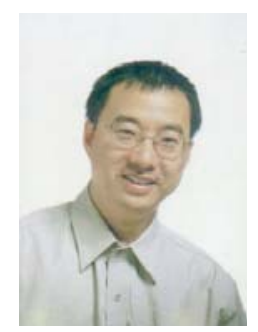

Kui Wu received the Ph.D degree in computing science from the University of Alberta, Canada in 2002. He then joined the Department of Computer Science, University of Victoria, Canada, where he is currently an Assistant Professor. His research interests include mobile and wireless networks, sensor networks, network performance evaluation, and network security.

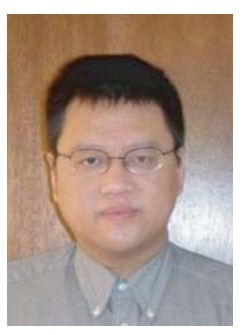

Yang Xiao is with Department of Computer Science, University of Alabama, USA. Dr. Xiao is an IEEE Senior Member. He was a voting member of IEEE 802.11 Working Group from 2001 to 2004. Currently, he serves as Editor-in-Chief for International Journal of Security and Networks, International Journal of Sensor Networks, and International Journal of Telemedicine and Applications. He has published more than $200 \mathrm{journal} / \mathrm{conference}$ papers. His research has been supported by NSF. Email: yangxiao@ieee.org.

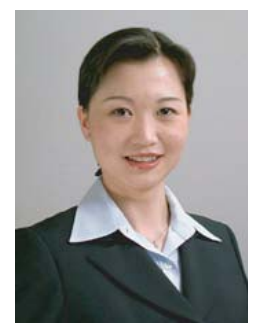

Jiang Xie received her B.E. degree from Tsinghua University, Beijing, China, in 1997, M.Phil. degree from Hong Kong University of Science and Technology in 1999, and M.S. and Ph.D. degrees from Georgia Institute of Technology in 2002 and 2004, respectively, all in electrical engineering. She is currently an assistant professor with the Department of Electrical and Computer Engineering at the University of North Carolina-Charlotte. Her current research interests include resource and mobility management of wireless networks, QoS provisioning, and next-generation Internet. She is a member of IEEE and ACM. 